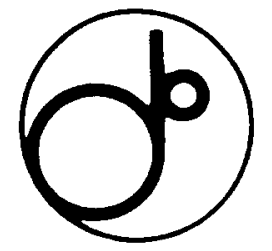

KEK Preprint 95-55

May 1995

A

\title{
Beam Test of a Button-type Beam-position Monitor for the ATF Damping Ring
}

F. HINODE, H. HAYANO, M. TEJIMA, N. TERUNUMA and J. URAKAWA
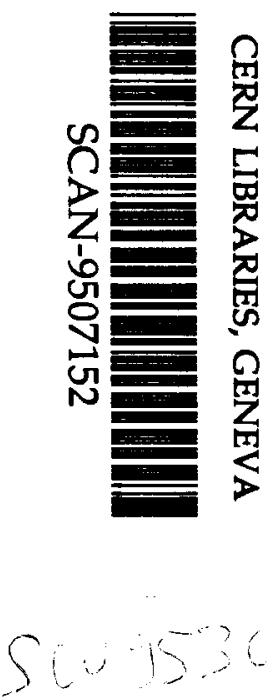

Presented at the 1995 Particle Accelerator Conference and International Conference on High-Energy Accelerators, May 1 - 5, 1995, Dallas, Texas, U.S.A. 
National Laboratory for High Energy Physics, 1995

KEK Reports are available from:

Technical Information \& Library

National Laboratory for High Energy Physics

1-1 Oho, Tsukuba-shi

Ibaraki-ken, 305

JAPAN

Phone: 0298-64-5136

Telex: $\quad 3652-534$ (Domestic)

(0)3652-534 (International)

Fax: $\quad$ 0298-64-4604

Cable: $\quad$ KEK OHO

E-mail: Library@kekvax.kek.jp (Internet Address) 


\title{
BEAM TEST OF A BUTTON-TYPE BEAM-POSITION MONITOR FOR THE ATF DAMPING RING
}

\author{
F.Hinode, H.Hayano, M.Tejima, N.Terunuma and J.Urakawa \\ KEK, National Laboratory for High Energy Physics, 1-1 Oho, Tsukuba-shi, Ibaraki-ken, 305 Japan
}

Button-type beam-position monitors (BPMs) were fabricated for the ATF damping ring. The BPM was designed to achieve a position resolution of less than $5 \mu \mathrm{m}$; fabrication of the first 40 BPMs has been completed. For this BPM, a beam test was carried out at the $80-\mathrm{MeV}$ injector part of the ATF LINAC. All of the bunch signals in the multi-bunch beam were clearly observed without any discharge. A calibration of the BPMs was also performed in order to check their offset from the electrical center to the mechanical center as well as their position detection sensitivity. The result shows good uniformity in position detection

\section{INTRODUCTION}

The ATF [1] is under construction at KEK in order to study the feasibility for the linear-collider (JLC [2]). The ATF damping ring will be operated at $1.54 \mathrm{GeV}$ with a multi-bunch electron beam, which will have a the vertical emittance of $5 \mathrm{x}$ $10^{-11} \mathrm{~m} \cdot \mathrm{rad}$. To achieve such a low-emittance beam we must correct for the dispersion of the orbit, which is small $(\eta<2$ $\mathrm{mm}$ ) in the long wiggler section of the damping ring. A precise measurement of the dispersion is indispensable. Usually, the actual dispersion is obtained by comparing each closed-orbit distortion under the conditions of different RF frequency $\left(\Delta \mathrm{f}^{\mathrm{RF}} \sim 10 \mathrm{kHz}\right.$ ). For this reason, the requirement for the resolution of the BPM is less than $5 \mu$ in [3]. Furthermure the impedance of the components, such as the racuum chamber, can be the source of a single-bunch instability which could degrade the beam quality. To avoid such an instability. the total longitudinal impedance must be less than about $0.2 \Omega$ [1]. The results of wake-field calculations indicate that the impedance is very small on the electrode of the butun-type: compared with the directional-coupler type [4] We therefore selected the button-type electrode for the BPM [5]

\section{CONFIGURATION OF THE BEAM POSITION MONITOR}

\section{A. Pickup Electrode}

The feedthrough consists of a central conductor with a button electrode, an outer conductor with an SMA connector and an insulator, as shown in Figure 1. The central conductor is made of Kovar and the button electrode is made of stainless steel. Taking into account the impedance, the curvature of the button shape is the same as the inner radius of the BPM block. The insulator is ceramic $\mathrm{Al}_{2} \mathrm{O}_{2}$. The outer conductor with the SMA connector is made of aluminum alloy with utanium joined by an HIP transition. Some electrodes have been tested

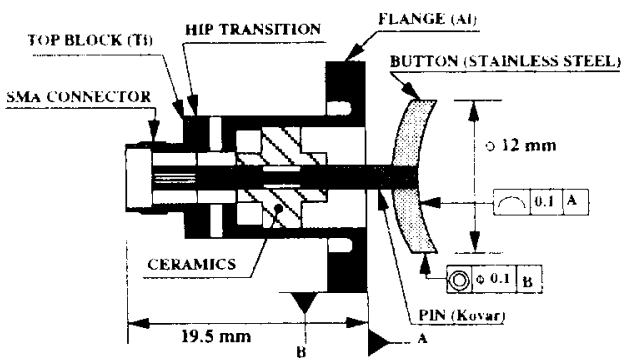

Figure 1: Pickup electrode for the BPM

for mechanical strength and vacuum leakage. The threshold level of the brazing strength of the central conductor is $60 \mathrm{~kg}$ against the tensile forces. No vacuum leak has occurred after heat-cycle tests from liquid-nitrogen temperature up to 200 C. Furthermore, all of the electrodes have been checked concerning their dielectric strength (> $1000 \mathrm{Volts}$ ).

b. BPM Block

Fach BPM is installed at a location near to every quadrupole magnet and sextupole magnet; there are 120 BPMs in the ring. We have already fabricated 40 BPMs in 1994

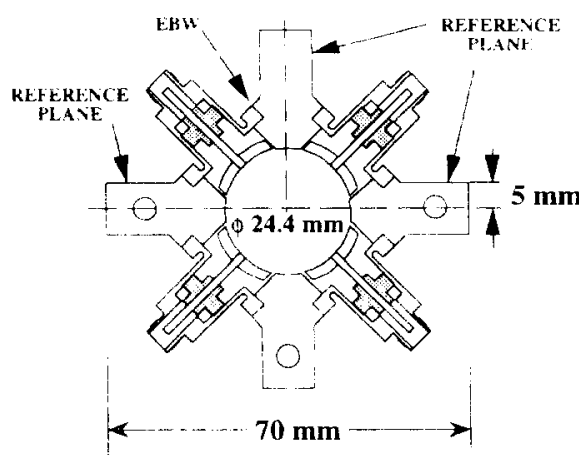

Figure 2: Cross-sectional view of the BPM

The BPM block was machined from a mass of aluminum alloy: four pickup electrodes were welded onto the block by electron beam welding (Figure 2). It has both horizontal and vertical reference planes, which are used for aligning the BPM to the $\mathrm{Q}$ magnet. Both planes are also used as a reference for the calibration process to obtain the electrical center of the BPM block. After installation of the vacuum chamber with BPMs to the beam line, the offset of the BPM to the Q magnet will be measured with an accuracy within $50 \mu \mathrm{m}$ by using the reference planes 


\section{BEAM TEST}

To study a low-emittance beam in detall, each bunch signal in the multi-bunch beam should be clearly distinguished. Furthermore, the BPMs must be operated stably against $3 \times 10^{10}$ particles per bunch at maximum. Since the feedthrough of the BPM has a cavity structure, there is a possibility that a discharge would occur due to the multipactering effect caused by resonance fields excited by the wake-field, which are induced by the beam. At the TRISTAN main ring, in fact, such a discharge effect. caused by the $\mathrm{TE}_{11}$ coaxial mode, was observed in a huttun-type BPM placed in the RF cavity section $(6$ ). The result it a resonance measurement shows that the lowest resonarice frequency is $11 \mathrm{GHz}$, which is consistent with the result calculated by the MAFIA code. According to the calculation of the multipactering zone [7]. at such a high frequency. although a discharge due to the multipactering will nor occur. it should be checked by an actual beam to check the performance of the BPM a beam test was carried out at the 80 $\mathrm{MeV}$ injector part of the ATF LINAC

\section{A. Setup of the Beam Test}

The setup of the beam test shown in ligure 3 Hesmen beams generated at the thermionc gun are accelerated up to $80 \mathrm{MeV}$, and then traverse the wall current monitur WCM. and the BPM. The BPM was welded to beam ducts having. length of $70 \mathrm{~mm}$ and an inner dianeter of $24 \mathrm{~mm}$, and places upstream of the beam dump. The output signal from the BPM is transmitted by RG-213/u cah/. $(-33 \mathrm{~m}$ ) and sberved by sampling oscilloscope (Tektrorix 11802. sampling head SI). 24). The output signal is attentated hefore the oscillusiofe due to the small dynamic range of the osclloscope. The beain current is measured by the WCM. The beam size and the bunch length were measured by a wire-scanner and a streak camera using an optical transition radiation as $02.2 \mathrm{rmm}$ and 20 psec in FWHM, respectively

\section{B. Beam Test using a Single-bun ti Bean}

An observed signal under single bunch operatuon is shown in Figure 4. The peak pulse height is $5 \mathrm{~V}+\mathrm{w} 18 \times 10$

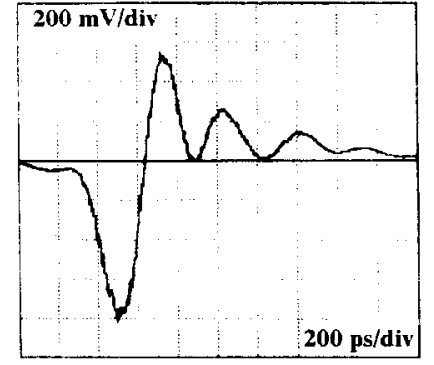

Figure 4: Observed signal of a single-bunch beam.

partucles. There is a ringing shape at the signal tail. This is caused by multiple reflections of the wall current at the edge of the BPM chamber due to the discontinuity. The difference in the inner diameter between the vacuum chamber of the I.INAC $(\$ .58 \mathrm{~mm})$ and the BPM $(\phi 24 \mathrm{~mm})$ is $34 \mathrm{~mm}$. However, the inner diameter is the same in the damping ring. Therefore, if mighi not be a problem for actual use. Furthermore, a prckup signal variation for the number of particles was also measured up to $2 \times 10^{10}$ particles per bunch, which showed goud linearity No discharge, such as multipactering, was ubserved. A beam test with a higher intensity beam will be performed after beam commissioning of the beam-transport ine this fall

\section{Betmi Jest asing a Multt-bunch Beam}

Figure 5 shows an ubserved signal for a multi-bunch beam. The bunch spacing is $2.8 \mathrm{nsec}$. All of the bunch signals were clearly observed without any discharge. The signal is

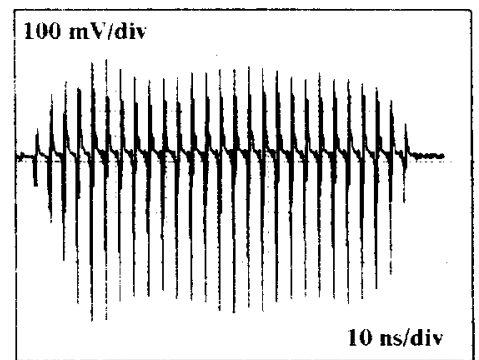

Figure 5 Observed signal of a multi bunch heam.

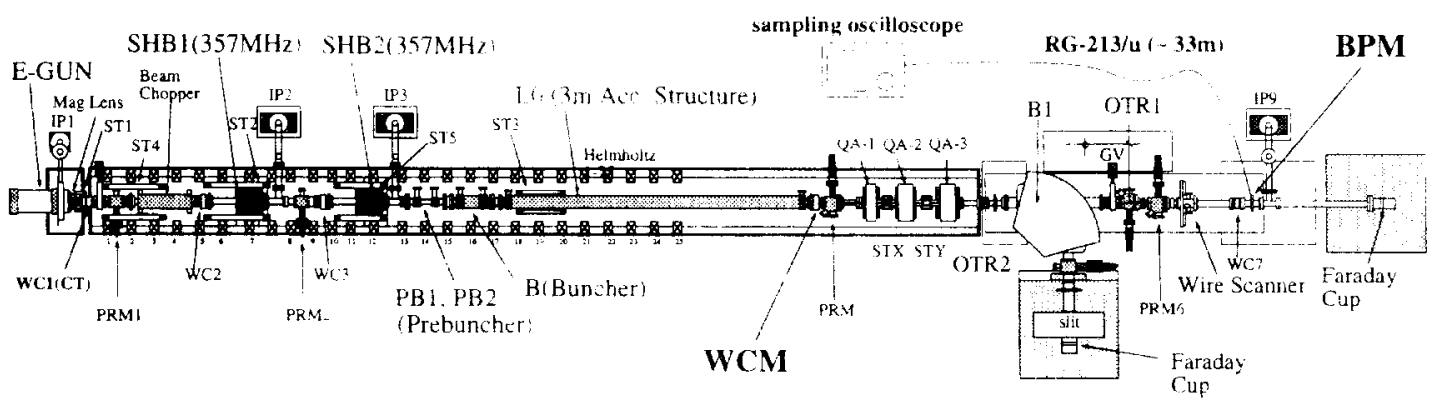

Figure 3 Setup of the beam test 
almost the same shape as that of the single-bunch beam. The signal tail of each bunch is sufficiently small when the next bunch signal arrives.

\section{Electrical Characteristics}

\section{A. Capacitance of Electrode}

Before and after electrodes were welded onto blocks, the capacitance of each electrode was measured using a capacitance meter at a frequency of $800 \mathrm{~Hz}$. The electrical. capacitance values are distributed in the $2.5 \sim 4.7 \mathrm{pF}$ region. Thus, four pickup electrodes with similar capacitance values were grouped and welded onto the monitor block in order to obtain uniform signals.

\section{B. Mapping for the Calibration}

Every BPM block was calibrated so as to obtain an offset between the mechanical and electrical centers of the BPM block $[1,5]$. The mechanical center is defined by the horizontal and vertical reference planes within the accuracy of machining. The electrical center was measured by the following procedure.

The BPM block was mounted on a fixed stage, and a 50 $\mu \mathrm{m}$ diameter tungsten wire was strung coaxially in the BPM block. Both ends of the wire were placed in a V-shaped groove made of ceramics, which was installed on both $x$ and $y$ movable stages. Each stage has a high positioning accuracy of $0.1 \mu \mathrm{m}$. One side of the wire was soldered to the SMA connector. The other end was terminated so as to match into a $50 \Omega$ line; attached $100 \mathrm{~g}$ weights provide a proper constant tension. The wire was put in the base position of a gauge which has the same reference planes as that of the BPM, and observed directly by a microscope. Thus, the wire was precisely aligned to the mechanical center of the BPM block with an accuracy of $40 \mu \mathrm{m}$. The calibration was performed by using a pulse signal with a 5 ns rise time. Signal processing is based on the same principle as that for the SLAC/FFTB [8] The readout electronics, which are a combination of a pulsestretcher amplifier, a Track\&Hold ADC and a pulse generator. have a resolution of $5 \mu \mathrm{m}$. We measured the four output signals $\left(\mathrm{V}_{1}, \mathrm{~V}_{2}, \mathrm{~V}_{3}, \mathrm{~V}_{4}\right)$ induced electrostatically on each electrode due to pulse signals transmitted on the wire. In uider to obtain the beam position, the following two calculation steps were performed. The first is a normalization procedure $\left(x^{\prime}, y^{\prime}\right)$, given by

$$
\mathrm{x}^{\prime}=k \frac{V_{2}-V_{4}}{V_{2}+V_{2}}, \mathrm{y}^{\prime}=k \frac{V_{1}-1}{V_{1}+V_{2}} .
$$

where $k$ is a coefficient of the sensitivity on the pusition measurement, which depends on the geometry of the monitor chamber. It was obtained by a measurement for each BPM Secondly, we converted the normalized results to the geometrical position $(x, y)$ according to

$$
x=\frac{\sqrt{2}}{2}\left(x^{\prime}-y^{\prime}\right), y=\frac{\sqrt{2}}{2}\left(x^{\prime}+y\right)
$$

In this way we obtained a relation between the measured position $(x, y)$ and the set position $(X, Y)$ of the wire position for each monitor.

The calibration was performed at 169 points in the central area, which is a $3.6 \mathrm{~mm}$ square region with a $0.3 \mathrm{~mm}$ step. As a result, there was no remarkable distortion in the mapping of all the BPMs. In the central region of $\pm 0.5 \mathrm{~mm}$ the distortion was less than $10 \mu \mathrm{m}$, while it was $100 \mu \mathrm{m}$ at a point $1.8 \mathrm{~mm}$ away from the center. This distortion is acceptable for our practical use. In the central region, however, it is only $2 \%$ and thus quite good.

We obtained the distribution of the offsets of the electrical center to the mechanical one from the calibration data. The mean values of the offsets are $\mathrm{X}=-19 \mu \mathrm{m}$ and $\mathrm{Y}=$ $58 \mu \mathrm{m}$, and the standard deviations are $-90 \mu \mathrm{m}$ in both directions. The main source of this deviation came from the errors in manufacturing the BPM block and pickup electrode.

The second fabrication of 40 BPMs and the calibration are now progressing.

\section{SUMMARY}

Button-type beam-position monitors (BPMs) were fabricated for the ATF damping ring. A beam test of the BPM was carried out at the $80-\mathrm{MeV}$ injector part of the ATF LINAC. All of the bunch signals in a multi-bunch beam were clearly observed without any discharge up to $2 \times 10^{10}$ particles per bunch. Calibration of the BPMs was also performed; the result shows good uniformity of position detection

\section{REFERENCES}

[1] ATF Design and Study Report, to be published as a KEK Internal Report

[2] JLC Group, KEK-Report 92-16.

[3] M. Tejima, Procs. of the SLAC/KEK Linear Collider Workshop on Damping Ring, KEK proceedings 92-6, 1992 , p. $126-132$

[4] M. Takao et al., KEK-Report 91-14.

[5] M. Tejima et al., Proceedings of the 1994 International Linac Conference, Vol. 2, pp. 914-916.

[6] M. Tejima et al., KEK Preprint 90-183.

[7] A. J. Hatch, Nucl. Instr. and Meth. 41 (1996) 261-271.

[8] H. Hayano et al.; KEK Preprint 92-118, H Hayano et al : SLAC-PUB-5691. 
